23

\title{
Оценка упорядоченности поперечной структуры мезопористых фотонных кристаллов
}

\author{
(C) Т.В. Миронова, А.В. Крайский \\ Физический институт им. П.Н. Лебедева РАН, 119991 Москва, Россия \\ e-mail: kraiski@sci.lebedev.ru
}

Поступила в редакцию 09.10.2020 г.

В окончательной редакции 22.12.2020 г.

Принята к публикации 30.12.2020 г.

\begin{abstract}
Оценивается однородность структуры мезопористого фотонного кристалла по электронной фотографии его поверхности. Предложен метод разбиения поверхности кристалла на домены с помощью корреляционной обработки цифрового изображения. В качестве основных численных характеристик упорядоченности системы пор рекомендованы следующие параметры: средний и максимальный линейные размеры домена, а также доля регулярных структур в образце. По этим и по ряду дополнительных параметров можно различать даже очень близкие по качеству структуры. Проведено сравнение изображений из различных источников.
\end{abstract}

Ключевые слова: алюминиевая фольга, фотонные кристаллы, поры, однородность структуры, цифровое изображение.

DOI: $10.21883 /$ OS.2021.04.50786.254-20

\section{Введение}

В настоящее время большое внимание уделяется исследованию, изготовлению и применению фотонных кристаллов. Фотонные кристаллы представляет собой среды, в которых диэлектрическая проницаемость периодически изменяется в пространстве. В последние годы был развит метод получения одномерных фотоннокристаллических пленок в результате электрохимического травления алюминиевой фольги [1-8]. В результате были получены мезопористые фотонно-кристаллические пленки анодного оксида алюминия, период которых по глубине слоя зависит от режима травления и может изменяться в диапазоне 100-500 nm [9]. При этом изменение показателей преломления $n_{1}$ и $n_{2}$ в смежных слоях фотонного кристалла осуществляется за счет различной степени пористости этих слоев, регулируемой в процессе анодного травления алюминиевой фольги в кислотной среде.

Пленки анодного оксида алюминия обладают рядом интереснейших не до конца изученных свойств. Например, было показано [2], что существует связь между поперечной и продольной упорядоченностью пор в пленках, в частности поверхностные домены с различной ориентацией пор содержат каналы с различными направлениями роста. Это делает изучение структуры поверхности пленки еще более актуальным. Множество работ, посвященных этому вопросу, можно условно разделить на два типа по методу подхода - локальный или глобальный. Исследования первого типа [1-3] базируются либо на получении диаграммы Вороного для исследуемого изображения и дальнейших попытках сделать выводы о качестве поверхности из структуры триангуляционной сетки, либо на измерении углов и расстояний между порами для всего изображения. Ко второму типу можно отнести работы, основанные на изучении фурье-спектров [4,5] и автокорреляционных изображений поверхности [6-8]. У каждого из подходов есть свои плюсы и минусы, в частности локальный подход требует удовлетворительного качества изображения и заметных вычислительных ресурсов. В качестве характеристики поверхности чаще всего выдается наибольший размер домена. Глобальный подход позволяет по форме пространственного спектра [4,5] или по соотношению высот пиков в автокорреляционном изображении [6] сделать общий вывод, какой из образцов лучше, а какой хуже. Также можно оценить средний размер пор и их степень эллиптичности [7].

Мы предлагаем своего рода комбинацию этих двух подходов. Предлагаемый метод позволяет обрабатывать как гексагональные, так и любые другие возможные текстуры с различной степенью регулярности. Благодаря использованию автокорреляции метод устойчив к небольшим дефектам и до некоторой степени к форме и размеру пор и чувствителен только к изменению структуры. Алгоритм прост и не требует больших ресурсов.

Таким образом, целью настоящей работы является исследование однородности свойств пористой структуры по поверхности и получение наиболее существенных численных характеристик степени ее упорядоченности, позволяющих сопоставлять по этим параметрам различные образцы с целью их оценки и оптимизации процесса травления.

\section{Результаты измерений и обсуждение}

Пример изображения поверхности фотонно-кристаллической пленки, полученного с помощью электронного 

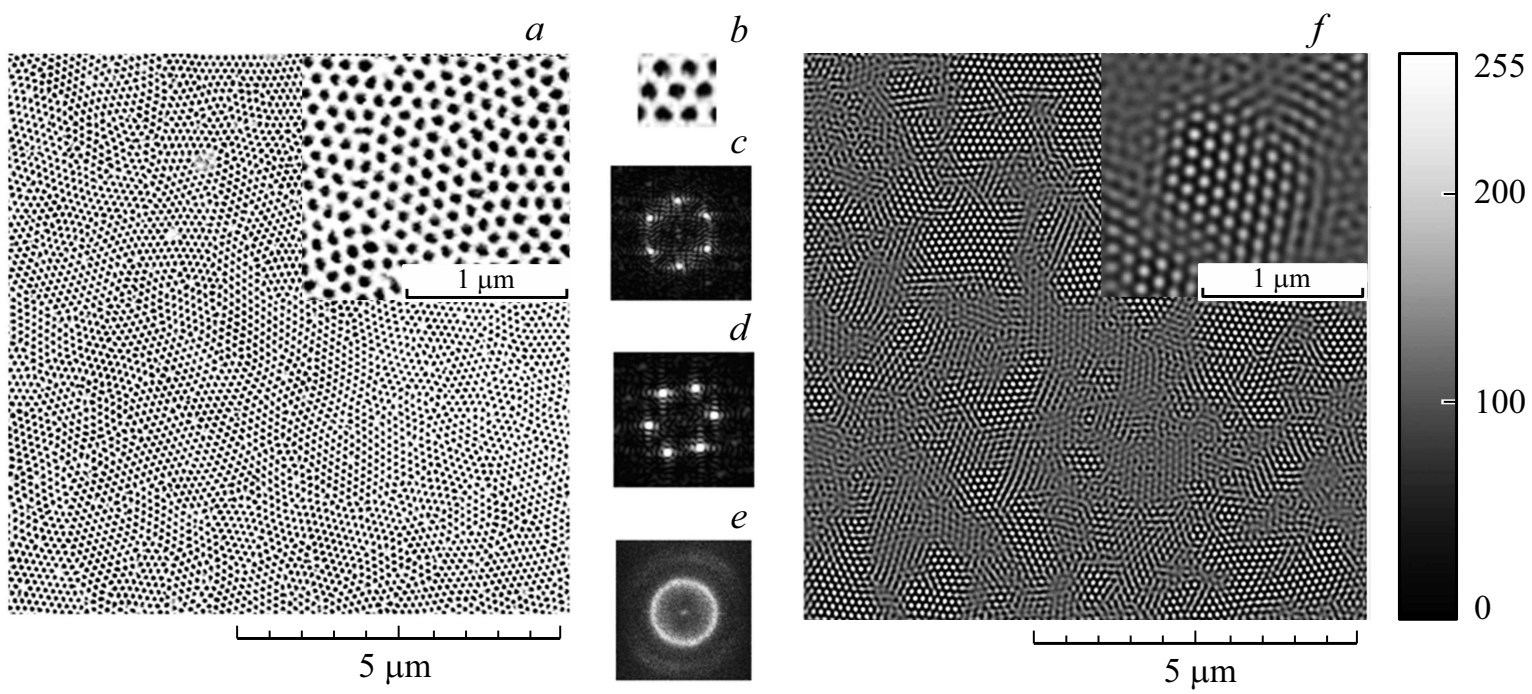

Рис. 1. Иллюстрация результата корреляционной обработки электронного изображения поверхности фотонно-кристаллической пленки. $(a)$ Исходное изображение поверхности. $(b)$ Часть изображения, взятая в качестве эталонного для одной из ориентаций структуры (увеличено). (c) Фурье-образ данного эталонного изображения. $(d)$ Фурье-образ эталонного изображения в другой ориентации. (e) Фурье-образ всего изображения, содержащего домены с различной ориентацией. $(f)$ Функция взаимной корреляции исходного и эталонного изображений.

микроскопа, показан на рис. 1,a [9]. Поры (диаметр $80 \mathrm{~nm}$, период $110 \mathrm{~nm})$, образованные в результате электрохимического травления алюминиевой фольги, достаточно плотно расположены по поверхности пленки. В то же время на приведенной фотографии заметно, что пористая структура упорядочена не по всей поверхности, а разбивается на своего рода „домены“ с различной ориентацией регулярной структуры. Также хорошо видны многочисленные дефекты в виде белых точек.

Для оценки однородности поверхности пленки и определения среднего размера „доменов“ мы применяем метод цифровой корреляции изображений [10-14]. Поскольку данный метод чувствителен к повороту объекта, корреляционной обработкой изображения можно выявить области с определенной ориентацией структуры расположения пор. Подобные измерения в аналоговой форме были проделаны в [15].

Пространственный фурье-спектр электронной фотографии изображения поверхности кристалла сам по себе содержит некоторую информацию о структуре ячеистой поверхности. Например, на рис. $1, c$ и $1, d$ показан фурье-спектр участков поверхности с различной ориентацией доменов. Поскольку в полном изображении содержатся домены различных ориентаций, его фурье-спектр будет своего рода суперпозицией локальных спектров (рис. 1,e). Неоднородность кругового сечения такого спектра позволяет оценить распределение ориентаций, а максимумы этого сечения - превалирующие направления ориентации доменов в изображении.

Пусть $I_{0}(\mathbf{r})$ - распределение интенсивности в полном исследуемом изображении, а $I_{1}(\mathbf{r})$ - эталонное изоб- ражение с определенной ориентацией структуры. Возьмем фурье-образы обоих изображений, а затем сделаем обратное фурье-преобразование от произведения этих фурье-образов $\Phi(\mathbf{q})=F_{0}(\mathbf{q}) F_{1}^{*}(\mathbf{q})$. При соответствующей нормировке такая процедура приводит к получению функции взаимной корреляции изображений

$$
K_{c c}(\boldsymbol{\tau})=\int_{S} d \mathbf{r} I_{0}(\mathbf{r}) I_{1}(\mathbf{r}+\boldsymbol{\tau}),
$$

где $S$ - область, по которой проводится интегрирование. В случае выбора на картине участка, являющегося копией эталона, смещенного относительно центра как целое без деформации и поворота, функция взаимной корреляции принимает вид

$$
K_{c c}(\boldsymbol{\tau})=\int_{S} d \mathbf{r} I_{0}(\mathbf{r}) I_{0}\left(\mathbf{r}+\boldsymbol{\tau}-\boldsymbol{\tau}_{0}\right),
$$

где $\boldsymbol{\tau}_{0}-$ вектор смещения. По положению максимума корреляционной функции определяется локальное значение смещения $\boldsymbol{\tau}_{0}$ для данной области интегрирования. Если в исследуемом изображении содержится не одно, а несколько эталонных изображений, функция корреляции будет иметь соответствующее количество локальных максимумов, имеющих соответствующие смещения. Распределение величины максимума функции взаимной корреляции по площади изображения для эталона с некоторой ориентацией системы пор (как на рис. 1,b) показана на рис. $1, f$. Она имеет вид квазипериодической двумерной функции с изменяющейся по координате относительной амплитудой модуляции. Участки корреляционной функции с большой амплитудой модуляции 
соответствуют областям исходного изображения с выбранной ориентацией системы пор (светлые участки изображения на рис. $1, f$ ). На врезках (рис. 1, $a$ и $1, f$ ) показан участок изображения, содержащий 1 домен, и функция корреляции эталонного элемента соответствующей ориентации с этим участком. Видно, что максимумы корреляционной функции расположены внутри границ домена. Форма огибающей корреляционной функции, т.е. степень локальности измерений, зависит от размера эталонного изображения (количества ячеек). Наиболее резкие контуры огибающей, как нетрудно понять, получаются при минимальном размере эталонного изображения, содержащего при этом достаточное число информационных элементов для уверенной работы корреляционного алгоритма (рис. $1, b$ ). В качестве базы для эталона можно принять либо участок поверхности, либо синтезированное изображение с идеальной геометрией. Исследования показали, что для нашей задачи это различие не играло принципиальной роли.

Корреляционная функция рассчитывалась для различных ориентаций эталонного изображения в диапазоне $0-55^{\circ}$ с шагом $5^{\circ}$. Доменом с заданной ориентацией считались области корреляционной функции, амплитуда модуляции в которых превышала 75\% от максимума. Этот выбор был обусловлен оптимальным совпадением конфигурации рассчитанных областей с визуально наблюдаемыми на фотографии. В число „хороших“ областей, т.е. областей с регулярной структурой какой-либо ориентации, при этом попадает 79\% всей площади. Если повысить порог отсечки корреляционной функции до $90 \%$, то в число регулярных областей не попадают не только явные дислокации, но и некоторые почти правильные, но слегка деформированные ячейки, а общая площадь „хороших“ областей занимает 64\% площади фотографии. Предполагается, что при выборе режима изготовления кристалла контрольные снимки будут одинакового качества, и тогда достаточно будет фиксировать режим обработки снимков для всей серии. Исходя из опыта работы для фотографий с другим разрешением или контрастом уровень отсечки корреляционной функции для идентификации регулярных структур можно выбирать в пределах 60-90\%.

Понятно, что в реальной ситуации углы поворота системы пор могут быть произвольными. Кроме того, чувствительность корреляционной функции к углу поворота не бесконечна [15]. Это приводит к тому, что рассчитанные конфигурации доменов для близких значений углов могут перекрываться. Степень перекрытия площадей рассчитанных доменов с различными углами ориентации иллюстрируется на рис. 2,a. По горизонтальной оси отложена разность углов ориентации структур. По вертикальной оси - степень перекрытия областей доменов. Для каждой пары ориентаций определялась площадь перекрытия рассчитанных областей, нормированная на общую площадь домена, а затем полученные данные усреднялись по всему ансамблю. В качестве ошибки на рисунке дано стандартное отклонение. Например, при разнице ориентации структуры в $5^{\circ}\left(0-5^{\circ}, 5^{\circ}-10^{\circ}\right.$, $10^{\circ}-15^{\circ}$ и т.д.) рассчитанные области перекрываются в среднем примерно на 50\%. При разнице углов $15^{\circ}$ и больше перекрытие становится пренебрежимо малым. Вышеизложенные соображения дают основания уменьшить дробность разбиения исходной картины на четыре типа ориентации структуры, обобщая данные в пределах пятнадцати градусов в одну общую картинку. То есть к одному типу областей относим области, полученные при ориентациях эталонного изображения для $-5^{\circ}, 0$ и $+5^{\circ}$ (обозначаем $0 \pm 5^{\circ}$ горизонтальная ориентация), $15^{\circ} \pm 5^{\circ}, 30^{\circ} \pm 5^{\circ}$ и $45^{\circ} \pm 5^{\circ}$. Результат такого разбиения показан справа на рис. 2 .

Различное направление штриховки обозначает рассчитанные домены с различной ориентацией структуры. Равномерно окрашенные серые области соответствуют местам дислокаций или границам доменов, где корреляционная функция ни для одной ориентации не достигла выбранного нами порога. При перекрытии рассчитанных доменов на границах (например, область с ориентацией $+5^{\circ}$ из первого объединенного домена перекрывается на $50 \%$ с областью $+10^{\circ}$, отнесенную нами ко второму домену) граница проводилась посередине полосы, относящейся к обоим объединениям.

На самом деле разбиение на отдельные домены процедура весьма условная. Между ними порою нет четких границ, и угол ориентации гексагональной структуры может плавно изменяться от точки к точке. Это явно видно на рис. $\mathrm{S} 1(b, d)$ в приложении к [2]. Нужен объективный численный критерий для сравнения. В качестве численной характеристики упорядоченности структуры предлагается вычислять наглядные числовые характеристики поверхности фотонного кристалла - общую долю регулярных структур, средний и максимальный линейный размер домена. Выбор линейного размера вместо, например площади домена, представляется нам более логичным, так как домены в общем случае могут представлять собой неодносвязные невыпуклые фигуры с сильно изрезанным краем.

Для иллюстрации работы метода выбраны три образца с гексагональной структурой поверхности. Образец № 1 [9] имеет субмикронную структуру не только по поверхности, но также и по глубине. Поперечный период структуры $138 \mathrm{~nm}$, диаметр пор $80 \mathrm{~nm}$. Образцы № 2 и № 3 [2] - это СЭМ-изображения нижней стороны мембран из анодного оксида алюминия, полученных на $\mathrm{Al}(100)$-подложке (№ 2) и на вицинальной грани монокристалла алюминия, наклоненной на $4.1^{\circ}$ (№ 3). Период структур $102 \mathrm{~nm}$. Расчеты для всех образцов проводились при одинаковых параметрах: корреляционная функция вычислялась для 12 направлений с шагом $5^{\circ}$, в качестве значимых областей выбирались те, где величина максимумов корреляционной функции превышала $80 \%$. Полученные по образцам данные сведены в таблице. Образец № 1 очевидно отличается от образцов № 2 и № 3 по трем основным параметрам. У него на $20 \%$ меньше средний линейный размер домена, заметно 

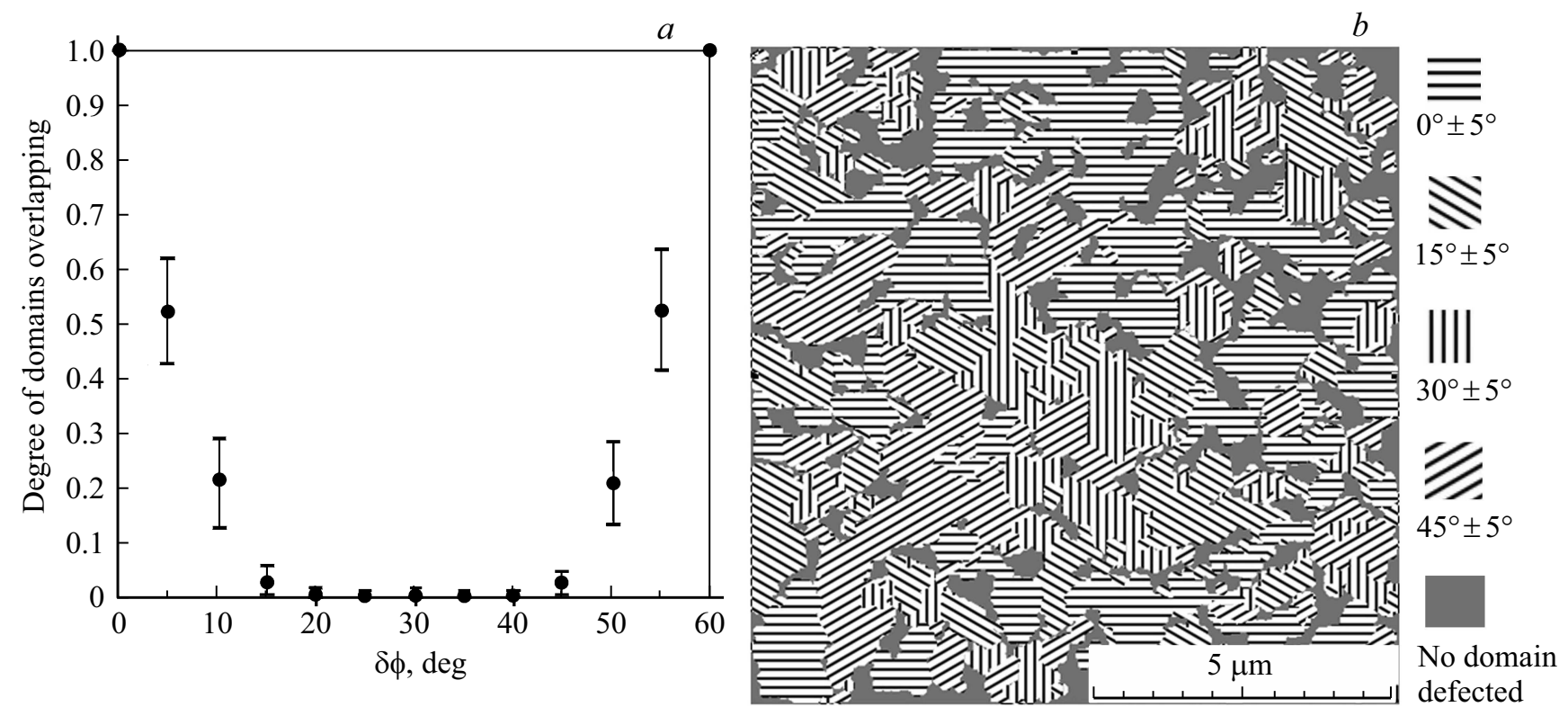

Рис. 2. Доменная структура. Доля перекрытия площадей доменов в зависимости от разницы углов ориентации структуры пор $(a)$. Разбиение изображения на домены $(b)$. Выбрано четыре направления: $\equiv$ - горизонтальное направление $\left(0^{\circ}-5^{\circ}\right)$, Nㅡㄴ $-15^{\circ}-5^{\circ}$, IIII $-30^{\circ}-5^{\circ}, \geqslant-45^{\circ}-5^{\circ}$. Ровно окрашенные серые области соответствуют нарушению структуры из-за дислокаций и на границах доменов.

\begin{tabular}{|c|c|c|c|c|c|c|c|c|}
\hline $\begin{array}{c}\text { Обра- } \\
\text { зец }\end{array}$ & $\begin{array}{c}\text { Средний линей- } \\
\text { ный размер } \\
\text { домена, } \mu \mathrm{m} \\
\text { и стандартное } \\
\text { отклонение }\end{array}$ & $\begin{array}{c}\text { Доля } \\
\text { регулярных } \\
\text { структур, \% }\end{array}$ & $\begin{array}{c}\text { Максимальный } \\
\text { линейный } \\
\text { размер домена, } \\
\mu \mathrm{m}\end{array}$ & $\begin{array}{c}\text { Максимальный } \\
\text { размер домена, } \\
\text { число ячеек }\end{array}$ & $\begin{array}{c}\text { Максимальная } \\
\text { площадь } \\
\text { домена, } \mu \mathrm{m}^{2}\end{array}$ & $\begin{array}{c}\text { Максимальная } \\
\text { площадь } \\
\text { домена, } \\
\text { число ячеек }\end{array}$ & $\begin{array}{c}\text { Средний раз- } \\
\text { мер домена } \\
\text { по одному } \\
\text { направлению, } \\
\text { максимум, } \mu \mathrm{m}\end{array}$ & $\begin{array}{c}\text { Средний раз- } \\
\text { мер домена } \\
\text { по одному } \\
\text { направлению, } \\
\text { минимум, } \mu \mathrm{m}\end{array}$ \\
\hline $1[9]$ & $\begin{array}{l}0.74 \\
0.05\end{array}$ & 89.7 & 3.6 & 26 & 13.9 & 833 & $\begin{array}{l}0.80 \\
\left(0^{\circ}\right)\end{array}$ & $\begin{array}{c}0.68 \\
\left(20^{\circ}\right)\end{array}$ \\
\hline $2[2]$ & $\begin{array}{l}0.93 \\
0.21\end{array}$ & 98.4 & 5.86 & 56 & 22.4 & 2485 & $\begin{array}{c}1.33 \\
\left(55^{\circ}\right)\end{array}$ & $\begin{array}{c}0.63 \\
\left(25^{\circ}\right)\end{array}$ \\
\hline $3[2]$ & $\begin{array}{l}0.95 \\
0.11\end{array}$ & 96.4 & 5.11 & 49 & 15.9 & 1767 & $\begin{array}{c}1.12 \\
\left(40^{\circ}\right)\end{array}$ & $\begin{array}{c}0.81 \\
\left(50^{\circ}\right)\end{array}$ \\
\hline
\end{tabular}

меньшая доля регулярных структур и максимальная площадь домена. С другой стороны, у образца № 1 значительно меньше относительный разброс линейных размеров доменов и значительно меньшая относительная вариабельность среднего размера по направлениям, т. е. этот образец более однороден по размерам доменов и более изотропен.

Различия между образцами № 2 и № 3 тоньше. Средний размер домена немного больше у образца № 3, но максимальный размер домена и доля регулярных структур больше в образце № 2. Большое стандартное отклонение при вычислении среднего размера и большая (более, чем в два раза) разница между средними размерами по выделенным направлениям дают основание утверждать, что в образце № 2 превалирует одна ориентация структур. Вычисления показывают, что домены с ориентацией $45^{\circ}-55^{\circ}$ занимают около $50 \%$ общей площади. Из этого можно сделать вывод, что структура образца № 2 более упорядочена.

\section{Заключение}

Показано, что с помощью корреляционной обработки электронных фотографий мезопористого кристалла можно оценить упорядоченность системы пор, получаемой при травлении. Предложен метод разбиения изображения поверхности кристалла на области с различающейся ориентацией системы пор, образованных при травлении алюминиевой фольги. В качестве основных численных характеристик упорядоченности системы пор предложены следующие параметры: средний и максимальный линейные размеры домена, а также доля регулярных структур в образце. Проведено сравнение изображений пленок анодного оксида алюминия как сильно различа- 
ющихся по степени упорядоченности, так и сравнимого качества. Основные и некоторые дополнительные численные параметры изображений представлены в таблице. Эти данные, по нашим представлениям, представляют интерес для выбора оптимальных режимов травления при изготовлении мезопористых фотонных кристаллов на основе анодного оксида алюминия.

\section{Конфликт интересов}

Авторы заявляют, что у них нет конфликта интересов.

\section{Список литературы}

[1] Gordeeva E.O., Roslyakov I.V., Napolskii K.S. // Electrochimica Acta. 2019. V. 307. P. 13-19.

[2] Roslyakov I.V., Koshkodaev D.S., Eliseev A.A. et al. // J. Phys. Chem. C. 2016. V. 120. P. 19698-19704.

[3] Hillebrand R., Muller F., Schwirn K., Lee W., Steinhart M. // ACS Nano. 2008. V. 2. P. 913-920.

[4] Michalska-Domańska M., Stȩnniowski W.J., Jaroszewicz L.R. // J. Porous Mater. 2017. V. 24. P. 779.

[5] Leitao D.C., Apolinario A., Sousa C.T. et al. // J. Phys. Chem. C. 2011. V. 115. P. 8567-8572.

[6] Pourfard M., Faez K., Tabaian S.H. // J. Phys. Chem. C. 2013. V. 117. P. $17225-17236$.

[7] Sacco L., Florea I., Châtelet M., Cojocaru C. // Thin Solid Films. 2018. V. 660. P. 213-220.

[8] Pichler S., Bodnarchuk M.I., Kovalenko M.V. et al. // ACS Nano. 2011. V. 5. N 3. P. 1703-1712.

[9] Горелик В.С., Яшин M.M., Dongxиe Bi, Guang Tao Fei. // Опт. и спектр. 2018. Т. 124. № 2. С. 12.

[10] Tropea C., Yarin A.L., Foss J.F. Springer Handbook of Experimental Fluid Mechanics. Berlin: Springer, 2007.

[11] Боркова В.Н., Крайский А.В, Миронова Т.В., Султанов Т.T. // Краткие сообщения по физике ФИАН. 2006. № 7. C. 38-41; Borkova V.N. et al. // Bull. Lebedev Phys. Inst. 2006. N 7. P. $38-41$.

[12] Крайский А.В., Миронова Т.В. // Измерительная техника. 2011. № 5. C. 26-29; Kraisky A.V., Mironova T.V. // Meas. Technol. 2011. N 5. P. 26-29.

[13] Крайский А.В., Кудрявцев Е.М., Миронова Т.В. // Краткие сообщения по физике ФИАН. 2012. № 9. С. 18-24. Kraiskii A.V., Kudryavtsev E.M., Mironova T.V. // Bull. Lebedev Phys. Inst. 2012. V. 39. P. 257.

[14] Kraisky A.V., Mironova T.V. How to Use a Digital Camera as a Metering Device. 2016. SPIE. SPOTLIGHT V. SL22. P. 48. doi $10.1117 / 3.2256725$

[15] Зубов В.А., Крайский А.В., Султанов Т.Т. // Краткие сообщения по физике. 1980. № 7. С. 24-30. 